\title{
Slow time scales in a dense vibrofluidized granular material
}

\author{
Andrea Plati ${ }^{1}$ and Andrea Puglisi ${ }^{2}$ \\ ${ }^{1}$ Dipartimento di Fisica, Università di Roma Sapienza, P.le Aldo Moro 2, 00185 Roma, Italy \\ ${ }^{2}$ Istituto dei Sistemi Complessi - CNR and Dipartimento di Fisica, \\ Università di Roma Sapienza, P.le Aldo Moro 2, 00185, Rome, Italy
}

(Dated: August 4, 2020)

\begin{abstract}
Modeling collective motion in non-conservative systems, such as granular materials, is difficult since a general microscopic-to-macroscopic approach is not available: there is no Hamiltonian, no known stationary densities in phase space, not a known small set of relevant variables. Phenomenological coarse-grained models are a good alternative, provided that one has identified a few slow observables and collected a sufficient amount of data for their dynamics. Here we study the case of a vibrofluidized dense granular material. The experimental study of a tracer, dispersed into the media, showed the evidence of many time scales: fast ballistic, intermediate caged, slow superdiffusive, very slow diffusive. A numerical investigation has demonstrated that tracer's superdiffusion is related to slow rotating drifts of the granular medium. Here we offer a deeper insight into the slow scales of the granular medium, and propose a new phenomenological model for such a "secular" dynamics. Based upon the model for the granular medium, we also introduce a model for the tracer (fast and slow) dynamics, which consists in a stochastic system of equations for three coupled variables, and is therefore more refined and successful than previous models.
\end{abstract}

\section{INTRODUCTION}

Granular materials stand as prototypes of physical systems with both important industrial applications and fundamental theoretical challenges [1, 2]. When the external perturbation is absent or very weak (and/or sparse in time), a granular medium behaves as an athermal amorphous solid: in this regime, theoretical approaches are scarce and can rarely be compared quantitatively with experiments $[3,4$. The situation is different in the case of so-called vibrofluidized regime, i.e. when a continuous external perturbation is applied, for instance by means of vibrating the box that contains the grains, provided that maximum shaking accelerations is much larger than gravity acceleration [5, 6. Actually such a regime can be separated into several different phases, depending upon the amount of grains in the container (typically measured through the number of layers at rest, or the average density/packing fraction) and the shaking parameters 7 , 8. In the most dilute and agitated phase, the so-called granular gas, quantitative predictions are obtained through granular kinetic theory [2] and granular hydrodynamics 9, 10. Those are bottom-up theories where macroscopic/average quantities, such as the transport coefficients, can be deduced from the knowledge of the laws of interaction among the single grains, with the assumption (true in the dilute Grad-Boltzmann limit) of molecular chaos, or its revised version called Enskog approximation [2, 9, 11 13. In the last decades, kinetic theory of molecular systems has made important progresses towards the quantitative understanding of the liquid phase [14, 15, with success in explaining certain aspects of slow relaxations in supercooled liquids 16, 17. A similar approach has been applied to vibrofluidized granular systems in order to obtain some predictions in non-dilute phases [18, 19]. This approach - based upon a granular adaptation of mode coupling theory (MCT) 17.
- reproduces the qualitative behavior of the relaxation of density correlations in the system, with the possibility of marking a glass transition where relaxation times diverge: at the qualitative level everything appears similar to the molecular (non-dissipative) case. The diffusional properties of a tracer are also qualitatively similar to molecular liquids, with the standard ballistic $\rightarrow$ arrested $\rightarrow$ diffusive scenario for the mean squared displacement. Rheological properties and the typical thinning-thickening scenario are also fairly explained with this approach, even if experiments may offer more complex pictures [20.

Experiments in dense vibrofluidized granular liquids in a cylindrical geometry (see Fig. 1) have shown the existence of a complex dynamical scenario with collective phenomena occurring along time-scales larger than the cage time-scale [21, 22]. The cylindrical geometry enhances the possibility of observing such time-scales: a cylinder implies the existence of a direction of motion the rotational one - with an infinite horizon, i.e. without obstacles (only subject to friction). The slow collective dynamics determines two observable phenomena: the superdiffusion (SD) for the dynamics of a tracer immersed in the granular medium [21, and persistent rotational motion (PR) of large parts of the granular medium [22]: both phenomena - strictly correlated - appear when the density is increased and/or the steady vibro-fluidization is reduced, and take place over timescales in the range of $10 \div 10^{3}$ seconds, depending upon packing fraction and shaking parameters, while interactions occur on timescales of $10^{-3} \div 10^{-2}$ seconds. Interestingly these unprecedented SD and PR are superimposed to the usually observed fast phenomena occurring over small and medium timescales, such as sub-collisional ballistic motion and transient dynamical arrest due to caging. Such kind of persistent motion is not accounted for by the aforementioned granular mode coupling theory, since velocities are factored out in that theory. 


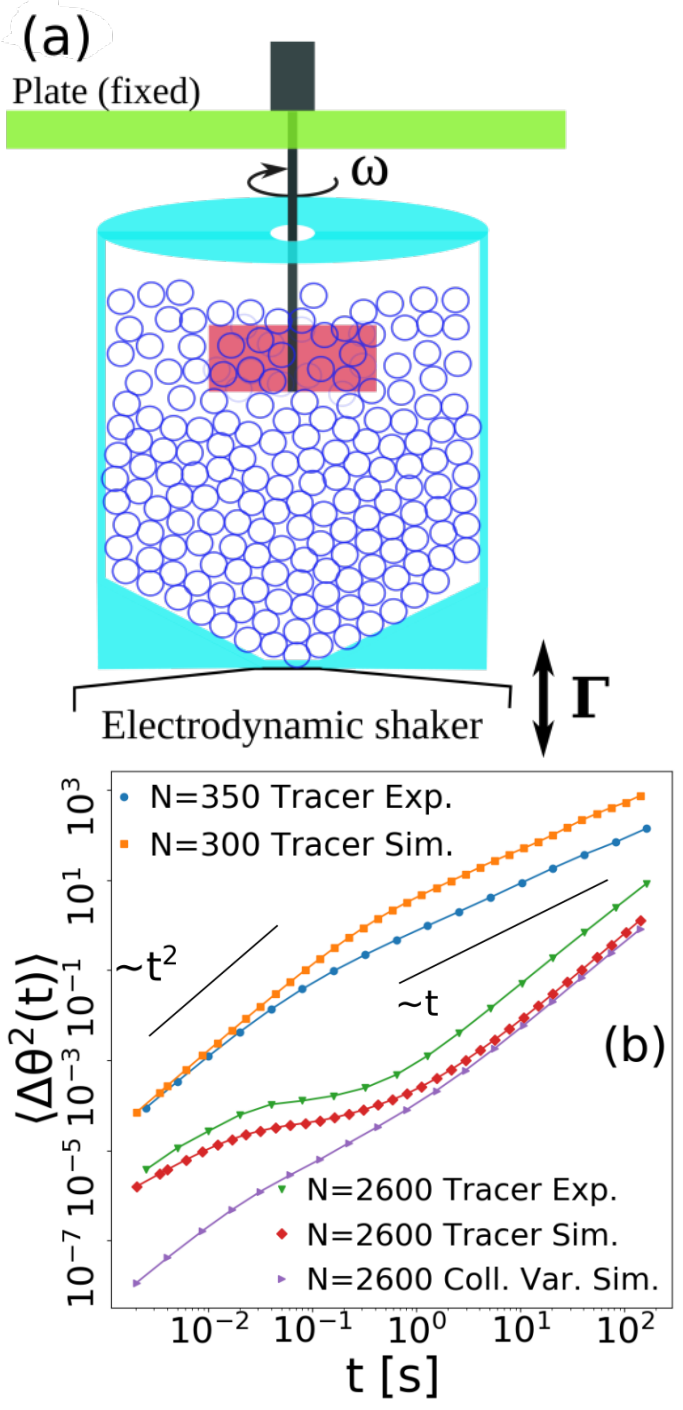

Figure 1. A: Setup of the experiment and of the simulation. B: mean squared displacements of the tracer (in experiments and simulations) and of the collective rotation of the granular medium (only simulations, see Section IV for the definition). $\Gamma=39.8$ for all the cases shown.

The rotational experiments are not unique - in the context of granular materials - to show some kind of collective motion occurring over very long time-scales. A long history of slow convective phenomena exists in regimes where the external vibration is exceedingly weak (below the gravity threshold) [23, 24, but we consider those cases substantially different from the case considered here, since they display basically only long timescales (no fast phenomena are present). More recently, a series of vibro-fluidized experiments has revealed the so-called "low frequency oscillations" LFO [25, 26], with frequencies below $1 \mathrm{~Hz}$, superimposed to standard fast relaxational behavior. $\mathrm{LFO}$ and $\mathrm{SD} / \mathrm{PR}$ phenomena occur over different ranges of frequency (LFO close to $1 \mathrm{~Hz}$, $\mathrm{SD} / \mathrm{PR}$ close to $0.1 \mathrm{~Hz}$ ) and their connection is still to be analyzed.

It is also interesting to compare the slow collective motion seen in these granular experiments with similar phenomena observed in models and experiments with active matter, for instance in dense populations of bacteria [27, 28] and sperms [29], or even within the dynamics of a single flagellum 30 .

In this paper, with the help of discrete-element simulations reproducing the original experiment in [21], we aim at elucidating some phenomenological stochastic models with few coarse-grained variables which are able to describe the SD and PR phenomena 31]. These models are an extension and improvement of a previous one proposed in 32 which was built to reproduce only the SD tracer behavior: limitations of that model are that it is not entirely coherent with the observed tracer power spectra and does not describe the granular medium (i.e. it cannot account for PR). These models share minimality (in the spirit of Occam razor), in fact they include only linear couplings and additive independent noises, allowing for an exact analytical treatment: their goal is to characterize the existence of many timescales in the system and for this purpose non-linearity is not a crucial ingredient. A more general (not limited to linear coupling) Langevin approach has been also considered by applying a Langevin modeling recipe to the experimental data [33]. Linearly coupled models have been used before in granular modeling: for instance they are able to characterize the failure of fluctuation-dissipation relation for the dynamics of a tracer in certain models [34, 35], but have shown certain limitations when compared with experiments 36]. Linear modeling used for the purpose of quantifying non-equilibrium features have been also used, recently, in the single-flagellum dynamics of active particles 30 .

The manuscript is organized as follows. In Section II we give a brief account of the existing stochastic models for this particular granular setup. In Section III the ingredients of the simulations are explained. In Section IV we show how the collective rotation of the granular medium is well reproduced by the superposition of two independent collective variables obeying linear Langevin equations with well-separated timescales. In section $\mathrm{V}$ we build upon the previous observation and describe the motion of a rotating tracer as a third rotating variable coupled to the granular medium, resulting de facto in a three-variable model which shows a close adherence to experimental and numerical power spectra. In Section VI we make a more extensive comparison of the two models with numerical and experimental data in order to rationalise the dependence of the models' parameters upon the physical parameters of the system. A more general discussion of the salient features of the proposed models, together with conclusive perspectives, is given in Section VII. 


\section{EXISTING LANGEVIN MODELS}

A simple and old model of diffusion in dense liquids is the so-called Itinerant Oscillator model, where the tracer is caged in a (harmonic) potential well, whose minimum's position is not fixed but slowly diffuses [37, 38]: this model helped in rationalising spectra from neutron scattering experiments on liquids [39. If diffusion of the potential minimum is slower than the particle's diffusion inside the well, then the behavior of the tracer's mean squared displacement (MSD) shows a transient plateau (equivalent to the dynamical arrest or caging) followed by ordinary diffusion. If the dynamics inside the well is underdamped, the first part of the tracer's MSD timedependence is ballistic $\sim t^{2}$. The after-cage part, however, is always of the ordinary diffusive type $\sim t$ as the tracer is slaved to the dynamics of the minimum's position which is purely diffusive. The extension of this model proposed in [32] was aimed at obtaining long superdiffusive regimes after the cage time. In order to obtain this result, an underdamped dynamics was considered also for the position of the well's minimum. Such an extension takes the form of four coupled equations for the angular velocity and position of the rotating tracer, $\omega(t)$ and $\theta(t)$ respectively, and for the angular velocity and position of a collective slow variable which represents the effect of a large group of particles surrounding the tracer (the cage), $\omega_{0}(t)$ and $\theta_{0}(t)$ respectively:

$$
\begin{aligned}
I \dot{\omega}(t) & =-\gamma \omega(t)-k\left[\theta(t)-\theta_{0}(t)\right]+\sqrt{2 \gamma T} \eta(t) \\
I_{0} \dot{\omega}_{0}(t) & =-\gamma_{0} \omega_{0}(t)+k\left[\theta(t)-\theta_{0}(t)\right]+\sqrt{2 \gamma_{0} T_{0}} \eta_{0}(t) \\
\theta(t) & =\int_{0}^{t} \omega\left(t^{\prime}\right) d t^{\prime} \quad \theta_{0}(t)=\int_{0}^{t} \omega_{0}\left(t^{\prime}\right) d t^{\prime} .
\end{aligned}
$$

In the above equations $\eta(t)$ and $\eta_{0}(t)$ are independent Gaussian white noises with zero average and unitary variance, namely $\left\langle\eta(t) \eta\left(t^{\prime}\right)\right\rangle=\delta\left(t-t^{\prime}\right)$ and $\left\langle\eta_{0}(t) \eta\left(t^{\prime}\right)\right\rangle=$ $\delta\left(t-t^{\prime}\right)$. The parameters $I$ and $I_{0}$ are the inertia of the tracer and of the surrounding medium, $\gamma$ and $\gamma_{0}$ are the dissipation felt by the two variables, and $T$ and $T_{0}$ are "temperatures" (assuming unitary Boltzmann constant $k_{B}=1$ ), see [35] for a discussion of their physical interpretation. The coupling between the tracer and the collective variable, in this model, is represented by the term $-k\left[\theta(t)-\theta_{0}(t)\right]$ which is linear in the positions. With large enough values of $I_{0}$, the inertia of the cage, the model - which is analytically solvable - reproduces long time ballistic superdiffusion.

A problem of this model however is its inability to entirely reproduce the velocity power density spectrum (PDS) which is put in evidence in Fig. 2, We recall the definition used here for velocity power spectrum

$$
S(f)=\lim _{t_{T O T} \rightarrow \infty} \frac{1}{2 \pi t_{T O T}}\left|\int_{0}^{t_{T O T}} \omega(t) e^{2 \pi f i} d t\right|^{2},
$$

(where $t_{T O T}$ is the total simulation time) which is



Figure 2. Comparison of the tracer's velocity power spectra (PDS) in experiments/simulations and the ones predicted (just for the experiments) by Eqs. (1) and (3). The two models fail in reproducing the data in region (II) in the same way. Both experiments and simulations are performed with $N=2600$. The peak close to $100 \mathrm{~Hz}$ seen in the experimental spectrum is directly related to the driving frequency and internal mechanical resonances of the setup, see 21] for details.

also equivalent to the Fourier transform of the autocorrelation function in the steady state $\langle\omega(t) \omega(0)\rangle$. The experimental/numerical power spectrum - read from high frequencies to low frequencies - shows four main regions (labels are marked in Fig. 2): IV) at very high frequency a power-law decay (slower than the pure Lorentzian case $\sim f^{-2}$ expected for exponentially decaying velocity autocorrelations), III) a bump-like peak at smaller frequencies representing the almost periodic oscillations inside a cage, II) a plateau at even smaller frequencies (suggesting a range of timescales where the process rapidly loses memory) and finally I) at the smallest observable frequencies (unless day-long experiments are conducted) a decay analogous to the high-frequency one. This decay at very small frequencies is in fact interpreted, in the above model, as the high-frequency decay of a very slow Ornstein-Uhlenbeck process described by $\omega_{0}(t)$. The most evident discrepancy between theory and experiments/simulations is in the central plateau region (II): the inertial itinerant oscillator model is not able to reproduce it.

A second attempt to obtain a meaningful Langevin model for the angular velocity of the rotating tracer has been done in 33. Its advantage is that it is the result of a general constructive method of Markovian model-building based upon the experimental data (the experimental time series of $\omega(t))$ and some guess about other possibly relevant variables (when data present nonMarkovian character, other variables must be identified in order to obtain a proper Markovian embedding [31]). In such a method one is also able to verify that the chosen variables are consistent with the Markovian hypothesis. Such an advantage results in a more compact model, with 
a smaller number of parameters:

$$
\begin{aligned}
\dot{\omega}(t) & =-A_{1} \omega(t)-A_{2}\left[\theta(t)-\theta_{0}(t)\right]+\sqrt{2 B} \eta(t) \\
\dot{\omega}_{0}(t) & =-A_{0} \omega_{0}(t)+\sqrt{2 B_{0}} \eta_{0}(t) \\
\theta(t) & =\int_{0}^{t} \omega\left(t^{\prime}\right) d t^{\prime} \quad \theta_{0}(t)=\int_{0}^{t} \omega_{0}\left(t^{\prime}\right) d t^{\prime},
\end{aligned}
$$

where $\eta(t)$ and $\eta_{0}(t)$ are Gaussian noises with unitary variance. This model is a particular limit of the model guessed in [32], where the term $k\left[\theta(t)-\theta_{0}(t)\right]$ is negligible with respect to the other terms in Eq. (1) b: indeed with the present definition, $\omega_{0}$ evolves with a slow dynamics that does not admit fluctuations on the fast time scale of $\theta_{1}$, so that such term is necessarily negligible. All other parameters are strictly related to the parameters of the model in Eq. 1. $A_{0}, A_{1}, A_{2}, B$ and $B_{0}$ should be directly compared with $\gamma_{0} / I_{0}, \gamma / I, k / I, \gamma T / I^{2}$ and $\gamma_{0} T_{0} / I_{0}^{2}$ respectively. This model well reproduces the mean squared displacement of the tracer with its final superdiffusive part, while its comparison with the power spectrum is unsatisfying in the central part, as in [32, see Fig. 2 ,

Models in Eqs. (1) and (3) both involve two independent white noises but they are anyway a threedimensional linear model in which the Markovian vector is $X(t)=\left\{z(t), \omega(t), \omega_{0}(t)\right\}$ with $z(t)=\theta(t)-\theta_{0}(t)$. One may wonder if it is possible to obtain satisfying results with a 2-variable linear model. We know from the data that a minimum request is a PDS with two stationary points (a minimum and a maximum) for $f>0$. In the appendix we show that this kind of functional form is not compatible with the most general form for a two-variable linear model. In view of this, using a three variable model is an unavoidable choice if one wants to reproduce the experimental data through stochastic linear models.

\section{MICROSCOPIC MODEL}

Simulations of the granular experiments are implemented through the LAMMPS package with its module dedicated to granular interactions [40]. LAMMPS is an optimized package that solves the molecular dynamics equations of motion and in our case incorporates the interactions of discrete elements methods to treat macroscopic (non molecular) particles such as the spherical grains of the experimental setup. Specifically all interactions among the bodies in the simulation, including interactions with the boundaries representing the experimental box, obey the Hertz-Mindlin model [4143]: the latter is a soft potential that takes into account normal and tangential forces, both made of elastic and dissipative contributions. Thus, our particles are spheres of mass $m_{i}$, radius $R_{i}$ and momentum of inertia $I_{i}=\frac{2}{5} m_{i} R_{i}^{2}$ with spatial coordinates $\vec{r}_{i}$ that travel with translational velocities $\vec{v}_{i}$ and rotational velocities $\vec{\omega}_{i}$. We specify that the flat boundaries of the box are considered as spheres with infinite mass and radius. When centers of mass of two particles are closer than the sum of their radii, a collision takes place, which is the only situation in which the interaction forces are non-zero: in that case particles $i$ and $j$ compenetrate to each other and a relative velocity at the surface of contact is defined as $\vec{g}_{i j}=\left(\dot{\vec{r}}_{i}-\vec{\omega}_{i} \times R_{i} \vec{n}\right)-\left(\dot{\vec{r}}_{j}+\vec{\omega}_{j} \times R_{j} \vec{n}\right)$ where $\vec{n}=\left(\vec{r}_{i}-\vec{r}_{j}\right) /\left|\vec{r}_{i}-\vec{r}_{j}\right|$; we call $\vec{g}_{i j}^{N}$ and $\vec{g}_{i j}^{T}$ the two projections, respectively normal and tangential, to this surface of contact. Now the equations of motion read as:

$$
\begin{aligned}
& \dot{\vec{g}}^{N}=\vec{F}^{N} / m^{\mathrm{eff}} \\
& \dot{\vec{g}}^{T}=\frac{7}{2 m^{\mathrm{eff}}} \vec{F}^{T},
\end{aligned}
$$

where $m_{i j}^{\text {eff }}=m_{i} m_{j} /\left(m_{i}+m_{j}\right)$ while $\vec{F}_{i j}^{N}=\vec{n}\left(\vec{n} \cdot \vec{F}_{i j}\right)$ and $\vec{F}_{i j}^{T}=\vec{F}_{i j}-\vec{F}_{i j}^{N}$ are respectively the normal and tangential component of the force $\vec{F}_{i j}$ between the particles. Both these two contributions are made of an elastic and a dissipative term, tuned by coefficients that depend upon the properties of the specific modeled material. To be more compact, we avoid to write here the full form of these terms, reported and explained in detail in the supplemental material of our previous study 22. In the same reference it is possible to find also the specific numerical values used to tune our simulations. The simulation setup fairly mimics the experimental setup of 21] with all its specific components and materials as the cylindrical box with the conical base and the blade used as intruder. The good quantitative agreement between the experimental and the numerical observations for the intruder has been shown in 22. (see also Fig. 1 here). In the same paper simulations without the blade have also been performed, in order to study the collective motion of the granular medium, inaccessible in the experiments. These studies confirmed that the PR phenomenon displayed by the granular medium is not affected by the presence of the tracer. Nevertheless we specify here that, except when explicitly declared, all the data relative to the collective motion shown in this paper (see $\Omega$ defined in the next section) come from simulation performed without the blade. To conclude this section, we mention that during the present numerical study an error in the source code of LAMMPS has been found [44]. In particular, the tangential force during the collision was always applied at the surface of the particles (i.e. at a distance $R_{i}$ from the center) and this naturally lead to an unphysical resultant torque during contact. This error is actually critical from a physical point of view because it breaks the conservation of the total angular momentum expected in internal (sphere-sphere) interactions. Nevertheless, we have corrected the code and run all the simulations again verifying that this error does not affect our old and new results in a significant way. Moreover from the LAMMPS stable release of 5 June 2019 the granular module has been updated and the error fixed [45]. 


\section{COLLECTIVE VARIABLE (TWO TIME SCALES)}

The results presented in 22 demonstrate that the SD phenomenon displayed by the rotating tracer is a direct consequence of the $\mathrm{PR}$ phenomenon exhibited by the granular medium: while on small time-scales the rotating tracer has its own dynamics with short free flights and rapid bounces against the boundaries of a local cage of surrounding grains, on long time-scales the tracer is dragged by a persistent collective rotation of the surrounding medium. Such a medium rotation is measured through a global angular velocity defined as

$$
\begin{gathered}
\Omega(t)=\frac{1}{N} \sum_{i=1}^{N} \dot{\theta}_{i}(t) \\
\theta_{i}(t)=\arctan \left(\frac{y_{i}(t)}{x_{i}(t)}\right) \quad \dot{\theta}_{i}(t)=\frac{\left(\mathbf{r}_{i}(t) \times \mathbf{v}_{i}(t)\right)_{z}}{r_{i}^{2}},
\end{gathered}
$$

where $N$ is the number of granular particles, $\mathbf{v}_{i}$ is the velocity of particle $i$, and $\mathbf{r}_{i}$ is the position of particle $i$ with respect to a coordinate system such that the origin lies on the axis of rotational symmetry of the setup. The time-integral of the global angular velocity represents a global absolute angle

$$
\Theta(t)=\int_{0}^{t} \Omega\left(t^{\prime}\right) d t^{\prime} .
$$

Such a variable, when density is increased and vibrofluidization is weakened, exhibits long persistent drifts in both clockwise and anti-clockwise direction. This implies the appearance of super-diffusion for its mean-squared angle $\left\langle\Delta \Theta^{2}\right\rangle(t)=\left\langle\left[\Theta\left(t^{\prime}+t\right)-\Theta\left(t^{\prime}\right)\right]^{2}\right\rangle \sim t^{\beta}$ with $\beta>1$, as seen in Fig. 1. Conversely, the power spectrum of the global angular velocity $\Omega(t)$ shows a power-law decay at very low-frequency. We recall that ordinary diffusion $(\beta=1)$ at long times must correspond, in the power spectrum of the velocity, to a plateau at low frequencies. The low-frequency decay is the symptom of persistent motion at long time-scales.

In this Section we propose a new model for the dynamics of the collective granular rotation. The model is based upon the same principles applied before to the motion of the tracer, i.e. that persistent memory can be reproduced by considering (at least) an auxiliary slow variable. An analysis of the data coming from numerical simulations showed, however, that in this particular case the modeling is even easier.

The model we propose for the dynamics of $\Omega(t)$ is the sum of two independent variables, a fast and a slow one. This direct superposition is different from the model in 32 where the tracer's velocity is always (harmonically) coupled to the slow variable, even at small timescales. In that case the slow variable represents cage dynamics and the coupling describes the natural confining interaction between the cage and the tracer. However such a cage dynamics is not present in the collective rotation (no cage exists for the rotational mode of the whole granular medium) and therefore there is not a simple mechanism coupling fast and slow motion: at a first level of approximation we can consider them to be decoupled. We choose for simplicity two independent OrnsteinUhlenbeck $(\mathrm{OU})$ processes with two different characteristic times $\tau_{1}=I_{1} / \gamma_{1}$ and $\tau_{2}=I_{2} / \gamma_{2}$ and, in general, two different temperatures $T_{1}$ and $T_{2}$. In summary the model is described by:

$$
\begin{array}{r}
\Omega(t)=\Omega_{1}(t)+\Omega_{2}(t) \\
I_{1} \dot{\Omega}_{1}(t)=-\gamma_{1} \Omega_{1}(t)+\sqrt{2 T_{1} \gamma_{1}} \eta_{1}(t) \\
I_{2} \dot{\Omega}_{2}(t)=-\gamma_{2} \Omega_{2}(t)+\sqrt{2 T_{2} \gamma_{2}} \eta_{2}(t) .
\end{array}
$$

In fact the model can be rewritten with a smaller number of parameters: the only coefficients that count are $\tau_{i}$ and $q_{i}=T_{i} / I_{i}$ with $i=1,2$.

We are in the presence of a sum of two independent variables, therefore the PDS and the MSD are simply the sum of the two individual OU contributions:

$$
\begin{array}{r}
\left\langle\Delta \Theta^{2}\right\rangle(t)=2 q_{1} \tau_{1} t+2 q_{1} \tau_{1}^{2}\left(e^{-\frac{t}{\tau_{1}}}-1\right) \\
+2 q_{2} \tau_{2} t+2 q_{2} \tau_{2}^{2}\left(e^{-\frac{t}{\tau_{2}}}-1\right) \\
S(f)=\frac{q_{1} \tau_{1}}{\pi\left[1+\left(2 \pi f \tau_{1}\right)^{2}\right]}+\frac{q_{2} \tau_{2}}{\pi\left[1+\left(2 \pi f \tau_{2}\right)^{2}\right]} .
\end{array}
$$

Our idea is then to consider one of the two characteristic times much larger than the other $\left(\tau_{2} \gg \tau_{1}\right)$. To make clear now the meaning of the two variables, we expect the slow component of the collective variable, $\Omega_{2}$, to behave similarly to the filtered variable

$$
\Omega_{s}(t)=\frac{1}{\tau_{f}} \int_{t}^{t+\tau_{f}} \Omega\left(t^{\prime}\right) d t^{\prime},
$$

obtained with a moving average of $\Omega(t)$ over a time $\tau_{f}$ such that $\tau_{2} \gg \tau_{f} \gg \tau_{1}$. In order to verify this conjecture we proceed in two ways: first we try to fit the numerical MSD and PDS via Eqs. (8), then we show that the superdiffusive part at late times of the collective MSD coincides with the MSD of the filtered variable $\left\langle\Delta \Theta_{s}(t)^{2}\right\rangle$ where $\Theta_{s}(t)=\int_{0}^{t} \Omega_{s}\left(t^{\prime}\right) d t^{\prime}$.

In Fig. 3 we show how Eqs. (8) can fit the numerical data for two particular cases of control parameters $(N=2600, \Gamma=19.5-59.8)$, postponing a more systematic analysis to section VI. In order to obtain the theoretical lines we have first performed a fit of the PDS via Eq. $8 \mathrm{~b}$ and then used the parameters inferred in this way also for the MSD (Eq. (8)a). We can see that the model properly predicts the behaviour of the two observables. In the PDS there is a good agreement at all the frequency regimes except for the high frequency decay where a linear model can only predict a $f^{-2}$ behavior while the data show $f^{-\alpha}$ with $2>\alpha>1$. The MSD also exhibits an almost perfect agreement between data and model predictions at all time scales. We conclude that the idea of decomposing the total collective 



Figure 3. Collective motion. First comparison between model predictions and numerical data $(N=2600, \Gamma=19.5-59.8$ ). MSD and PDS refer to the same signal. The simple model in Eqs. (7) fits well the numerical data for both the PDS and the MSD. The fitted parameters are reported in Table I.

variable into two independent contributions that act at two well-separated time scales is reasonable. Looking at the numerical values of the fitted parameters in Table I one can can verify that $\tau_{2} \sim t_{T O T} \gg \tau_{1}$.

In Fig. 3 $3 \mathrm{~b}$ we show the MSD of $\Omega$ and $\Omega_{s}$ for two values of $\Gamma$, one cold case and one hot case at similar density. For the cold case, i.e. at low $\Gamma$, we see that $\left\langle\Delta \Theta(t)^{2}\right\rangle$ is ballistic at all times except for the initial ones (the fast component is very weak so the slow one emerges immediately). In the warmer case we can clearly distinguish the two contributions to the total MSD: the fast one that dominates the first times with its ballistic part and then diffuse, and the slow one that dominates the late times with superdiffusion. Regarding the filtered MSDs we can see that in both cases it emerges in the total one exactly at the beginning of the superdiffusive regime, at late times. In principle it is not obvious that the filter used for $\Omega_{s}$ is able to isolate the slow component of a sum of two signals. We can clearly expect that $\left\langle\Delta \Theta_{s}(t)^{2}\right\rangle$ has to overlap the total MSD for $t>\tau_{f}$ but how can we be sure that it is really describing the MSD of $\Omega_{2}(t)$ i.e. the slow component of $\Omega$ ? We try to answer to this question with Fig. 4 where we study the effect of the filter on two qualitatively different signals for several choices of $\tau_{f}$. We first discuss the MSD of the collective variable in a dilute case $(\mathrm{N}=300)$ where we have an ordinary ballistic-diffusive behavior with just one relevant time scale [22]. We can see that in this case the filter lowers the energy of the ballistic part and stretches the ballistic part up to times $t \sim \tau_{f}$ where the filtered MSD reunites with the original one. In the dense case (e.g. low $\Gamma$ ) the MSD has the ballistic-diffusive-superdiffusive behavior with two relevant time scales (the fast $\tau_{1}$ and the slow $\tau_{2}$ of the aforementioned model) but we can consider a third one $\tau^{*}$ defined as the time for which the slow variable starts to dominate the total MSD (so the time when the late superdiffusion starts, close to $\tau_{2}$ ). In this case the filter acts as in the previous one but for $\tau_{f}>\tau^{*}$ it reaches a kind of saturation and leaves the shape of the MSD unchanged (see the yellow and the cyan-dashed lines that overlap). This implies that for $\tau_{f}>\tau^{*}$ the time in which $\left\langle\Delta \Theta(t)^{2}\right\rangle$ and $\left\langle\Delta \Theta_{s}(t)^{2}\right\rangle$ reunite coincide with $\tau^{*}$ and no more with $\tau_{f}$. In view of these last analysis we can conclude that if in our data a contribution of a slow variable is present, this filter operation tends to isolate it.

To sum up, in this section we have provided evidences of two main facts:

- The collective variable behaves as the sum of two independent OU processes with different characteristic times $\Omega(t)=\Omega_{1}(t)+\Omega_{2}(t)$;

- A running average $\Omega_{s}(t)=\tau_{f}^{-1} \int_{t}^{t+\tau_{f}} \Omega\left(t^{\prime}\right) d t^{\prime}$ with $\tau_{f}$ larger than the time $\tau_{1}$ in which the fast component dominates, successfully isolates $\Omega_{2}(t)$ i.e. the slow component of $\Omega$.

\section{MOTION OF A ROTATING TRACER (THREE TIME SCALES)}

Once we have a satisfying model for the collective granular motion, a model for the tracer's motion can be studied on a solid basis, with the aim of improving the model in 32. The final model for the tracer appears as a system of three equations for three variables (where actually two of them are independent from each other). To build this new model we have considered that the tracer is moving in a complex granular fluid: such a fluid has two characteristic time scales, as explained in the previous section. The coupling between the tracer and the background granular fluid can be modeled in two different ways: as a conservative interaction that depends 


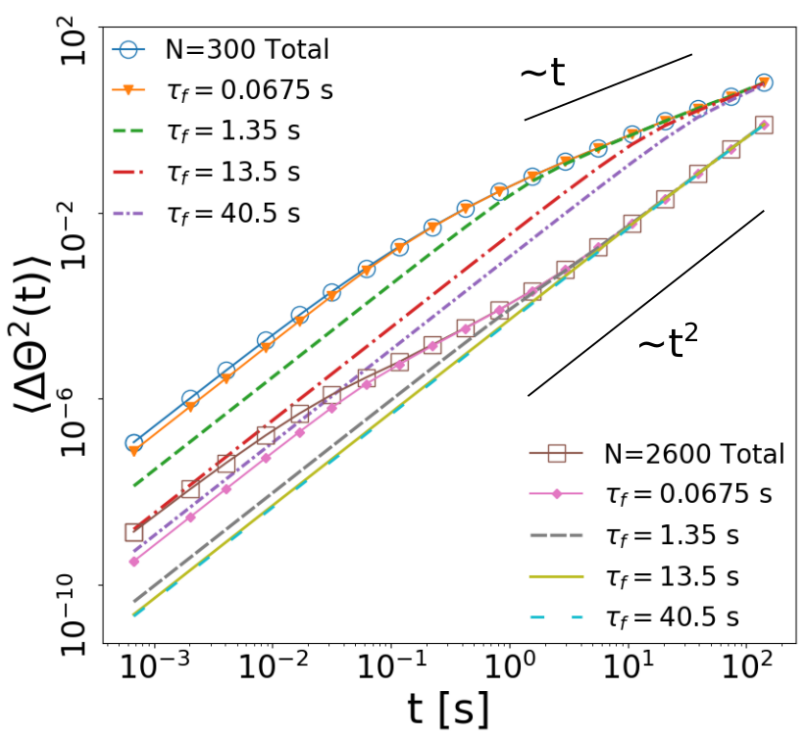

Figure 4. A: MSD of the total signals and the filtered ones for several $\tau_{f}$ s and two values of $N$ with fixed $\Gamma=39.8$.

upon a relative position between the tracer and a representative fluid particle, or as a viscous interaction that depends upon the relative velocity between the tracer and the fluid. The first choice was adopted in 32, but our present study convinced us that the second choice gives a better comparison with data. For a viscous interaction, we were inspired by [35] were a linear model for a massive granular tracer in a granular fluid (in a planar, not cylindrical, geometry) was considered. The tracer - characterized by velocity $V(t)$ - was coupled with the fluid characterized by local velocity $U(t)$ - through a viscous drag term proportional to $V(t)-U(t)$. Depending on the specific region of the parameter space, this model can reproduce both ordinary Brownian motion and cage effects with the ballistic-caged-diffusive behavior in the MSD and the back-scattering peak in the PDS. Therefore, it only lacks the superdiffusion at late times to properly describe the phenomenon under interest here. As we explained in section II, we cannot expect such a complex behavior from a linear model with two variables, therefore we have to insert a third one trying to be as less artificial as possible. To do so we leave the equation for the tracer unchanged and complicate the expression of the auxiliary variable making it coincide with the collective variable $\Omega$ relative to the sub-set of the granular particles in the bulk that actually influence the tracer dynamics. This quantity is always modeled by Eqs. (7) and defined by Eq. (5a) but with the mean operation extended just on the aforementioned sub-set of grains. We expect for it the same qualitative behavior (but in general not quantitative) of the global collective variable studied in the previous section: for this reason we decided not to introduce a new symbol for it.

We end up with a three-variable linear model defined

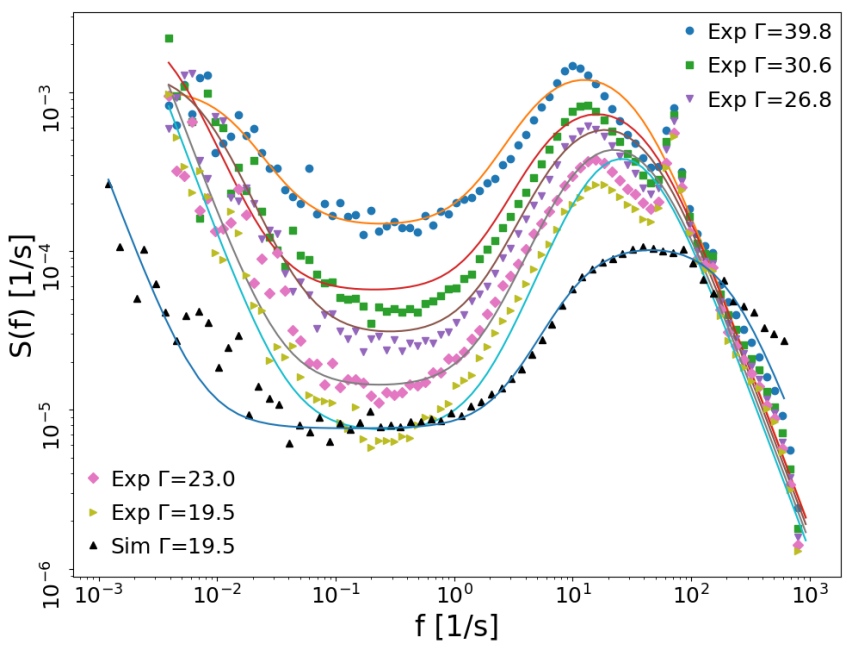

Figure 5. Comparison between experimental/numerical data and model (Eq. 10) for the PDS of the tracer for many values of $\Gamma$ and fixed $N=2600$. See caption of Fig. 2 for explanation of peak close to $100 \mathrm{~Hz}$ in the experimental spectrum.

by the following equations:

$$
\begin{array}{r}
I \dot{\omega}(t)=-\gamma(\omega(t)-\Omega(t))+\sqrt{2 T \gamma} \eta(t) \\
\Omega(t)=\Omega_{1}(t)+\Omega_{2}(t) \\
I_{1} \dot{\Omega}_{1}(t)=-\gamma_{1} \Omega_{1}(t)-\gamma_{c} \omega(t)+\sqrt{2 T_{1} \gamma_{1}} \eta_{1}(t) \\
I_{2} \dot{\Omega}_{2}(t)=-\gamma_{2} \Omega_{2}(t)+\sqrt{2 T_{2} \gamma_{2}} \eta_{2}(t) .
\end{array}
$$

Here we see that the bulk collective variable $\Omega$ acts as a local velocity field on the tracer whose velocity is $\omega(t)$. At the same time, the fast component of the collective variable $\Omega_{1}$ is coupled with $\omega$ through a viscous constant $\gamma_{c}$. These last two ingredients originate the observed broad cage effect. The slow component $\Omega_{2}$ is independent from the other variables and, as suggested by the numerical analysis, it will emerge at late times in the MSD and at small frequency in the PDS. Regarding the physical meaning of the coefficients we have for each variable an inertia $I_{i}$, a viscous coefficient $\gamma_{i}$ and a temperature $T_{i}$. We note that the dilute limit (simple OU process for the tracer) is recovered by sending $\gamma_{1} / I_{1} \rightarrow \infty$ and $\gamma_{2} / I_{2} \rightarrow \infty$ while the model for the collective variable alone (Eqs. 7) is obtained by setting $\gamma_{c}=0$. As already noted in previous studies [35, 46], we recall here that Eqs. (10) are equivalent to a Generalized Langevin Equation with exponential memory which is consistent with a typical approximation done for Brownian Motion when, at high densities, the coupling of the tracer with fluid hydrodynamics modes, decaying exponentially in time (see [47, Cap 8.6 and 9.1), must be taken into account.

From Eqs. 10), exploiting the formalism of the multivariate linear stochastic processes [48], we can compute (see Appendix) the PDS of the tracer. In Fig. 5 we show a comparison with the experimental and numerical data, finding good agreement in all the frequency regimes. The improvement with respect to the model defined by Eqs. 
(1) and (3) regards the form of the PDS. In the previous model, the peak and the valley of the PDS are specular i.e. their position and width depend upon the same combination of parameters so they cannot be changed independently (Fig. 2). The experimental and numerical PDS show instead that the valley and the back-scattering peak are never specular and in general this is coherent with the scenario suggested by the numerical simulations. Indeed, the valley is actually the crossover between the motion of the tracer inside the cage and the movement of the cage itself that enslaves the tracer. Once verified the presence of a persistent collective motion of the granular medium we can say that the cage moves as the collective variable on two time scales that are, with a good approximation, independent. In this picture it is thus reasonable that the frequency (time) where the slow component emerge in the PDS (MSD) could change independently from what is happening at the characteristic frequency (time) of the back-scattering (cage) effect and vice-versa. This is possible with Eqs. (10) by changing a combination of two parameters and leaving the others fixed (Fig. 6.).

Both for Eqs. (7) and (10) the number of independent parameters needed is actually smaller than the one used but we have kept some redundancy for the purpose of presenting more clear model equations. Now, rescaling all the equations by the inertia $I_{i}$ we can rewrite the two models in a compact form that is more suitable for reading the next section. For the tracer we have:

$$
\begin{array}{r}
\dot{\omega}(t)=-\frac{1}{\tau}(\omega(t)-\Omega(t))+\sqrt{2 \frac{q}{\tau}} \eta(t) \\
\Omega(t)=\Omega_{1}(t)+\Omega_{2}(t) \\
\dot{\Omega}_{1}(t)=-\frac{1}{\tau_{1}}\left(\Omega_{1}(t)+\alpha \omega(t)\right)+\sqrt{2 \frac{q_{1}}{\tau_{1}}} \eta_{1}(t) \\
\dot{\Omega}_{2}(t)=-\frac{1}{\tau_{2}} \Omega_{2}(t)+\sqrt{2 \frac{q_{2}}{\tau_{2}}} \eta_{2}(t) .
\end{array}
$$

where $\tau_{i}=I_{i} / \gamma_{i}, q_{i}=T_{i} / I_{i}$ and $\alpha=\gamma_{c} / \gamma_{1}$. Regarding the model for the collective variable alone we note that the rescaled form of Eqs. 77) (for the granular bulk dynamics when the tracer is absent) coincide with Eqs. (11p-d) once set $\alpha=0$.

The last aspect of the proposed model we want discuss regards the physical meaning of couplings between variables in system of Langevin equations. First we note that also without the introduction of ausiliary variables the effect on the tracer of the surrounding fluid is intrinsically contained in the Langevin approach. Indeed, also for ordinary Brownian motion (Eq. (11a) without $\Omega(t)$ ) the characteristic time $\tau$ and the stationary variance $q$ depend on the properties of both tracer and fluid [49]. The need of additional variables emerges just in presence of multiple time scales in the tracer dynamics. In view of this, referring to Eqs. 11, we can say that from an energetic point of view the effect of the granular medium on the tracer can be contained in $q$ alone. Indeed, provided that $\tau \ll \tau_{1}$ and $q \gg q_{1}$, the fluctations in the steady states of $\omega$ are not affected by the introduction of $\Omega_{1}$. We can expect that this limit holds in our system because the inertia of the local collective variable $I_{1}$ is reasonably higher than the one of the blade $I$. We will confirm this expectation in the next section in which our fitting procedure shows that $q$ and the variance of $\omega$ are almost coincident as in a single variable process.

These features are independent of $\alpha$ in Eq. (11c) so, in this limit, $\alpha$ represents the adimensional strength of a coupling that affects just the memory and not the energy of the tracer dynamics. In other words, the introduction of $\Omega_{1}$ changes the shape of the PDS of $\omega$ leaving its integral unaltered (we remember here that if $\langle\omega\rangle=0$ then $\left.\int_{0}^{\infty} d f S(f)=\left\langle\omega^{2}\right\rangle\right)$. Remarkably, studying the derivative of $S(f)$, it is possible to see that $\alpha>0$ is a necessary condition for the occurrence of the back-scattering effect. So, in our model, this effect is possible only if the tracer is coupled with a variable that is influenced by the tracer itself. This fact is compatible with the intuitive physical mechanism with which back-scattering is rationalized: the surrounding fluid is perturbed by the intruder and the latter feels with some delay in time the effect of this perturbation. In view of this mutual influence, we find even more appropriate the definition of $\Omega$ as a local field in Eqs. (10) and (11). Indeed, even if the whole granular medium can be reasonably unperturbed by the intruder, there will be always a local fraction of it that reciprocally interacts with the tracer givig rise to the back-scattering effect.

This clarifies also the way in which the tracer is coupled with $\Omega_{2}$ that is not affected by $\omega$. Indeed, the cage of surrounding grains has two main effects: confining the tracer with back-scattering (coupling between $\omega$ and $\Omega_{1}$ with reciprocal influence) and dragging it into the slow dynamics (coupling between $\omega$ and $\Omega_{2}$ without reciprocal influence).

\section{PHYSICAL MEANING OF THE MODEL'S PARAMETERS}

In this Section we attempt to systematically fit our model's parameters with the results of the numerical simulations and experiments. This task has two main motivations. First, it may suggest a way to infer or guess the model's parameters (or their behavior when physical parameters are varied) in general situations. Second, it makes more robust the identification of the model: a fuzzy or unintelligible behavior of the model's parameter would be the symptom of a weakness of the model itself.

For this purpose we report in Table I the fitted parameters obtained for the collective variable (simulations) via Eqs. $11 \mathrm{~b}-\mathrm{d}$ ) with $\alpha=0$ and for the tracer (experiments and simulations) via Eqs. (11) for many values of $\Gamma$. We first concentrate on the numerical data for the collective variable $\Omega$. From Eqs. (7) and (11) it is clear that the sum of two independent OU processes depends on four parameters: $\tau_{1}, q_{1}, \tau_{2}, q_{2}$ where $\tau_{i}$ is the characteristic 




Figure 6. Four different shapes of $S(f)$, obtained by plotting the PDS of $\omega(t)$ computed from model 10$)$ or different arbitrary choices of the parameters where $\tau_{i}=I_{i} / \gamma_{i}, q_{i}=T_{i} / I_{i}$ and $\alpha=\gamma_{c} / I_{1}$. We demonstrate that it is possible to change the broadness of the valley independently from the one of the back-scattering peak and vice-versa.

time of the single process and $q_{i}$ is its variance. In Section IV we verified that Eqs. (7) properly reproduce the functional form of the numerical PDS and MSD, now we want to study how the fitted parameters behave as a function of $\Gamma$. In particular, we are interested to verify if their numerical values reflect the physical intuitions on which the model with two variables is based. In Fig. 7 we can clearly see that $\tau_{1}$ and $q_{1}$ are increasing with $\Gamma$ while $\tau_{2}$ and $q_{2}$ are decreasing. The behavior of $q_{1}$ is intuitive because we can reasonably think that this parameter grows with the "temperature" of the physical external driving (the shaker). The behavior of $\tau_{2}$ and $q_{2}$ corroborates our intuition that reducing $\Gamma$ induces the emergence of a slow time scale whose persistency time $\left(\tau_{2}\right)$ and intensity $\left(q_{2}\right)$ grow. The microscopic origin of this fact can be understood by considering the dynamical heterogeneity present in this system (see for instance [22]): local temperature and pressure may vary a lot in space. When a collective motion emerges, we find that a great fraction of particles participates - as a condensed state - to the collective motion and a smaller one still exhibits a gas-like behavior. We can actually imagine that the slow contribution $\Omega_{2}$ to the total variable $\Omega$ is mainly due to the particles in this condensed phase, whose number could be thought as proportional to the effective inertia of $\Omega_{2}$. At this point it is clear that increasing $\Gamma$ reduces the fraction of particles in the condensed phase and consequently reduces $\tau_{2}$. The growth of $\tau_{1}$ with $\Gamma$ has not an easy explanation in our opinion. In dilute kinetic models the dissipative drag (here inverse of $\tau_{1}$ ) is often related to the mean collision frequency (mediated through a ratio of masses and other factors): however this quantity may have opposite trends when $\Gamma$ grows, i.e. it may increase because there
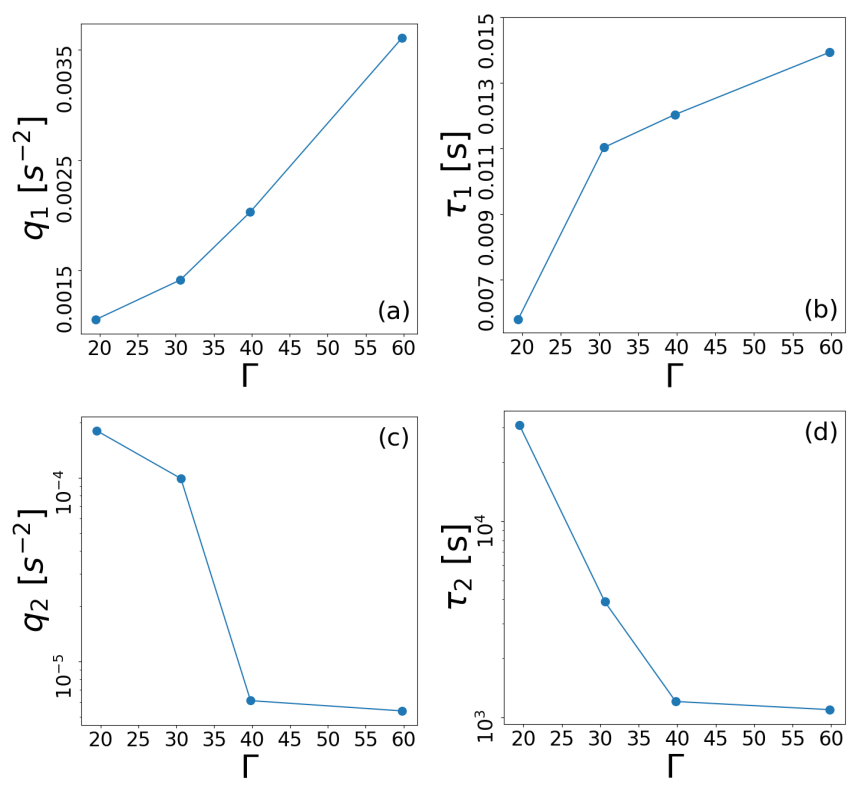

Figure 7. Fitted parameter for the collective variable (simulations) versus $\Gamma$ with fixed $N=2600$. Fit has been done via PDS (Eq. (8b ) relative to Eqs. $111 \mathrm{p}-\mathrm{d}$ ) with $\alpha=0$. Numerical values of the parameters are also reported in Table I.

is more energy (faster collisions) or it may decrease because there is a larger mean free path. Apparently the second phenomenon dominates the first. The connection between dissipation and collision frequency, however, is reasonable for dilute gases but certainly not obvious in condensed phases.

Regarding the experimental data of the tracer, from Eqs. (11) we see that the number of free parameters for a fit is 7 and they are $\tau, q, \tau_{1}, q_{1}, \tau_{2}, q_{2}, \alpha$. We recall that $q, q_{1}$ and $q_{2}$ would be equal to the stationary variances of $\omega, \Omega_{1}$ and $\Omega_{2}$, respectively, if these variables were not coupled. In our model with couplings one must consider the covariance matrix $\sigma$ (see Appendix A.2) which is related to the noise amplitudes through a relation which also involves the coupling matrix [48. However, in view of the discussion about couplings at the end of Sec. V, and with the aim of reducing the freedom in the fitting procedure, we decided to set $q$ to coincide with the variance of $\omega$ measured in experiments. We then verify $a$ posteriori how $\mathrm{g}$ ood is this approximation. In Fig. 8 we compare experimental $\left\langle(\omega-\langle\omega\rangle)^{2}\right\rangle$ with the theoretical one calculated with fitted parameters and verify that our assumption is reasonable. The behavior of $q$ together with the ones of $\tau, \tau_{2}$ and $q_{2}$ shown in the same figure (panels b, c and d), is coherent with the phenomenology already explained for fast and slow parts of the collective variable of Fig. 7 (we remind that $\Omega_{2}$ is totally independent from the other variables so $q_{2}$ always coincides with the variance of $\Omega_{2}$ ). Indeed, the idea of our model is to consider the tracer $(\omega)$ enslaved by the collective variable: at short times it feels the effect of the fast component $\Omega_{1}$ 


\begin{tabular}{|c|c|c|c|c|c|c|c|}
\hline & $\tau_{1}[\mathrm{~s}]$ & $q_{1}\left[\mathrm{~s}^{-2}\right]$ & $\tau_{2}[\mathrm{~s}]$ & $q_{2}\left[\mathrm{~s}^{-2}\right]$ & $\tau[\mathrm{s}]$ & $q\left[\mathrm{~s}^{-2}\right]$ & $\alpha$ \\
\hline$\Gamma=19.5$ Tracer Simulations & $9.6 \mathrm{e}-02$ & $5.7 \mathrm{e}-03$ & $1.1 \mathrm{e}+04$ & $2.0 \mathrm{e}-02$ & $7.3 \mathrm{e}-04$ & $4.4 \mathrm{e}-01$ & 5.0 \\
$\Gamma=19.5$ Coll. Var. Sim. with blade & $5.8 \mathrm{e}-03$ & $1.1 \mathrm{e}-03$ & $1.1 \mathrm{e}+04$ & $8.1 \mathrm{e}-04$ & $\backslash$ & $\backslash$ & $\backslash$ \\
$\Gamma=19.5$ Coll. Var. Simulations & $5.8 \mathrm{e}-03$ & $1.1 \mathrm{e}-03$ & $3.1 \mathrm{e}+04$ & $1.8 \mathrm{e}-04$ & $\backslash$ & $\backslash$ & $\backslash$ \\
$\Gamma=30.6$ Coll. Var. Simulations & $1.1 \mathrm{e}-02$ & $1.4 \mathrm{e}-03$ & $3.9 \mathrm{e}+03$ & $9.9 \mathrm{e}-05$ & $\backslash$ & $\backslash$ & $\backslash$ \\
$\Gamma=39.8$ Coll. Var. Simulations & $1.2 \mathrm{e}-02$ & $2.0 \mathrm{e}-03$ & $1.2 \mathrm{e}+03$ & $6.1 \mathrm{e}-06$ & $\backslash$ & $\backslash$ & $\backslash$ \\
$\Gamma=59.8$ Coll. Var. Simulations & $1.4 \mathrm{e}-02$ & $3.6 \mathrm{e}-03$ & $1.1 \mathrm{e}+03$ & $5.4 \mathrm{e}-06$ & $\backslash$ & $\backslash$ & $\backslash$ \\
\hline$\Gamma=19.5$ Tracer Experiments & $1.03 \mathrm{e}-01$ & $1.0 \mathrm{e}-03$ & $4.6 \mathrm{e}+03$ & $4.2 \mathrm{e}-01$ & $2.8 \mathrm{e}-03$ & $4.5 \mathrm{e}-01$ & 6.8 \\
$\Gamma=23.0$ Tracer Experiments & $1.06 \mathrm{e}-01$ & $1.1 \mathrm{e}-03$ & $7.0 \mathrm{e}+03$ & $5.1 \mathrm{e}-01$ & $2.8 \mathrm{e}-03$ & $5.1 \mathrm{e}-01$ & 5.0 \\
$\Gamma=26.8$ Tracer Experiments & $1.09 \mathrm{e}-01$ & $1.2 \mathrm{e}-03$ & $2.0 \mathrm{e}+01$ & $4.7 \mathrm{e}-03$ & $3.0 \mathrm{e}-03$ & $6.2 \mathrm{e}-01$ & 3.7 \\
$\Gamma=30.6$ Tracer Experiments & $1.10 \mathrm{e}-01$ & $1.4 \mathrm{e}-03$ & $4.0 \mathrm{e}+01$ & $3.4 \mathrm{e}-03$ & $3.3 \mathrm{e}-03$ & $7.3 \mathrm{e}-01$ & 2.8 \\
$\Gamma=39.8$ Tracer Experiments & $1.11 \mathrm{e}-01$ & $2.0 \mathrm{e}-03$ & $1.1 \mathrm{e}+01$ & $2.5 \mathrm{e}-03$ & $4.2 \mathrm{e}-03$ & $9.5 \mathrm{e}-01$ & 2.1 \\
\hline
\end{tabular}

Table I. Numerical values of the fitted parameters for the collective variable in simulations via Eqs. 10 b-d) with $\alpha=0$ and the tracer in experiments and simulations via Eqs. (11). The first and the second rows refer to the same simulation with the blade.

(high frequency decay and back-scattering peak) while at late times $\Omega_{2}$ starts to dominate the entire dynamics with its persistent ballistic drifts. The motion of the tracer is then characterized by more than one characteristic time scale. Looking at Eq. 10a it is quite natural to associate $\tau$ and $q$ respectively to the characteristic time and the variance of the short-time dynamics of $\omega$. It is therefore reassuring to find for these parameters similar behaviors to those observed for $\tau_{1}$ and $q_{1}$ in numerical simulations (compare panels a and b of Fig. 7 and 8 . Also the values of $\tau_{1}$ and $q_{1}$ obtained from the experimental data via Eqs. (11) (not shown in figures but reported in Table I) follow the same qualitative behavior. Regarding couplings, our fitting procedure revealed the situation depicted at the end of Sec. V. Looking at Table I and Fig. 8 we find that the effect on the tracer of the auxiliary variables is negligible form an energetic point of view $\left.\left(q \simeq(\omega-\langle\omega\rangle)^{2}\right\rangle\right)$ but not from a memory one $(\alpha \sim \mathcal{O}(1-10)$ for all the fitted spectra $)$.

The results reported in Fig. 8 regard the experimental tracer but, as is shown for one case $(\Gamma=19.5)$ in Fig. 5, we have also tested our model on the numerical data coming from the simulations with the blade. We note that the good agreement shown is obtained for values of $\tau_{1}, q_{1}, \tau_{2}$ and $q_{2}$ that are quite different from the ones coming from a direct fit of the two components of the collective variable in simulations with or without the blade (see first, second and seventh rows of Table I). This is not surprising because, as already stated at the end of section $\mathrm{V}$, the variable $\Omega$ actually coupled to the tracer, in Eqs.10 and (11), is a local collective variable. The latter reasonably differs - quantitatively - from the global one because of a considerable spatial heterogeneity of granular temperature and diffusivity 22.
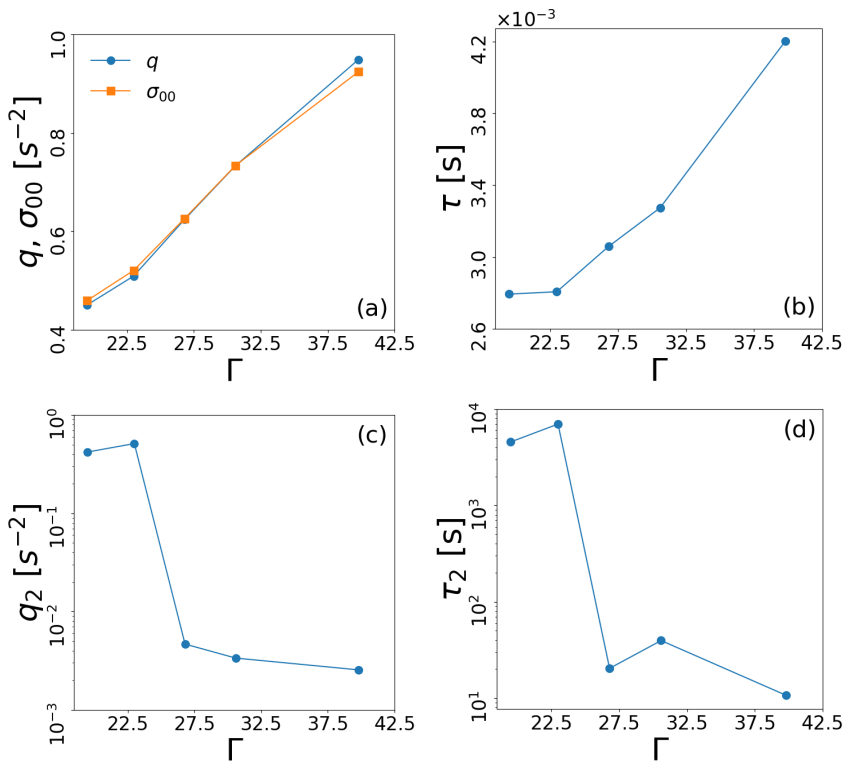

Figure 8. Fitted parameters for experimental tracer versus $\Gamma$ with fixed $N=2600$. Fit has been done via PDS (see Eq. (A7) in the Appendix) relative to Eqs. 111. Numerical values of the parameters are also reported in Table I.

\section{CONCLUSIONS}

In conclusion, we have proposed a series of linear stochastic models to rationalise a series of experimental and numerical results. In the phenomenon we have tackled, vibrofluidized dense granular materials display persistent slow drifts superimposed to fast collisional processes. We stress that the proposed models are purely phenomenological. They can be considered analogous to hydrodynamic models for dense fluids (even granular fluids), where the transport coefficients are not derived from microscopic parameters but are obtained from empirical observations. An important added value of phenomeno- 
logical models for slow variables is to offer arguments in favor of the idea of scale separations, which is not always guaranteed in granular fluids [33, 50, 51.

We have built two main models. The one for the motion of the angular drift of the granular medium, which is a sum of two independent Langevin diffusions (i.e. Brownian motion with inertia), characterizing fast and slow scales: the independence is consistent with the lack of cage effects which are usually an evidence of coupling between fast and slow scales. The presence of inertia also in the slow mode provides the main ingredient for persistent ballistic motion. The second model is for the motion of a rotating tracer immersed in the granular medium, which is the most accessible variable in experiments. Such a model is built upon the idea that the tracer is coupled with a local fraction of the surrounding medium through a purely viscous interaction, similar to other granular tracer models. Since both models contain several independent parameters, it is not surprising that they reproduce all available data. Less trivial is the fact that the fitted parameters behave in a coherent way and are consistent with physical intuition, as shown in Section VI. We have also solved an important inconsistency of a previous model for the tracer [32, which was unable to describe the power spectrum at medium time-scales.

We hope to stimulate future theoretical investigations in order to derive these models, or part of their parameters, following a kinetic theoretical approach.

\section{ACKNOWLEDGMENTS}

The authors are indebted to Marco Baldovin for fruitful scientific discussions. The authors acknowledge the financial support of Regione Lazio through the Grant "Progetti Gruppi di Ricerca" N. 85-2017-15257 and from the MIUR PRIN 2017 project 201798CZLJ.

\section{Appendix A: Multivariate Linear Stochastic Processes}

\section{Generic process with two variables}

A generic multivariate linear stochastic process can be written as $\dot{X}(t)=-A X(t)+B \tilde{\eta}(t)$, where $X(t)$ is a vector of variables, $\tilde{\eta}(t)$ a vector of white noises with zero mean and unitary variance while $A$ and $B$ are the matrices that define characteristic times, diffusion coefficients and eventually couplings of the variables. In two dimension, assuming independent noises between the variables, we have $X(t)=\left\{x_{0}(t), x_{1}(t)\right\}$ and:

$$
A=\left(\begin{array}{ll}
a & b \\
c & d
\end{array}\right), \quad B=\left(\begin{array}{cc}
D_{1} & 0 \\
0 & D_{2}
\end{array}\right), \quad \tilde{\eta}(t)=\left(\begin{array}{l}
\eta_{1}(t) \\
\eta_{2}(t)
\end{array}\right)
$$

We can now compute the spectral matrix through the following relation [48]:

$$
S(f)=\frac{1}{2 \pi}(A+i 2 \pi f)^{-1} B B^{T}\left(A^{T}-i 2 \pi f\right)^{-1}
$$

obtaining for the spectrum of a single variable (for example $\left.x_{0}(t)\right)$ :

$S_{00}(f)=\frac{\frac{1}{2 \pi} D_{1}^{2} d^{2}+2 \pi D_{1}^{2} f^{2}+\frac{1}{2 \pi} D_{2}^{2} b^{2}}{\left(16 \pi^{4} f^{4}+4 \pi^{2} f^{2}\left(a^{2}+2 b c+d^{2}\right)+a^{2} d^{2}-2 a b c d+b^{2} c^{2}\right)}$

Computing the first derivative we find $S_{00}^{\prime}(f)=$ $P(f) / Q(f)$ where $P(f)=c_{1} f^{5}+c 2 f^{3}+c 3 f$ with $c_{1}, c_{2}<$ 0 . This polynomial cannot have a double stationary point in the region $f>0$ so there is not any choice of parameters that reproduce the behavior under interest here i.e. a low-frequency decay followed by a back-scattering peak. These results does not change if we take $x_{1}(t)$ instead of $x_{0}(t)$.

\section{Model for The Tracer}

From Eqs. 10 we have $\dot{X}(t)=-A X(t)+B \tilde{\eta}(t)$ with $X(t)=\left\{\omega(t), \overline{\Omega_{1}}(t), \Omega_{2}(t)\right\}$ and:

$$
\begin{aligned}
A & =\left(\begin{array}{ccc}
\mu & -\mu & -\mu \\
\mu_{1} & \alpha & 0 \\
0 & 0 & \mu_{2}
\end{array}\right), \\
B & =\left(\begin{array}{ccc}
\sqrt{2 \mu q} & 0 & 0 \\
0 & \sqrt{2 \mu_{1} q_{1}} & 0 \\
0 & 0 & \sqrt{2 \mu_{2} q_{2}}
\end{array}\right), \\
\tilde{\eta} & =\left(\begin{array}{c}
\eta(t) \\
\eta_{1}(t) \\
\eta_{2}(t)
\end{array}\right) .
\end{aligned}
$$

where $\mu_{i}=1 / \tau_{i}=\gamma_{i} / I_{i}, q_{i}=T_{i} / I_{i}$ and $\alpha=\gamma_{c} / \gamma_{1}$. The covariance matrix $\sigma$ is realted to $A$ and $B$ through the relation: $A \sigma+\sigma A^{T}=B B^{T}$. We have computed the spectrum used for the fit of sec. V and VI always through Eq. (A2) obtaining Eq. (A7) where $\hat{f}=2 \pi f$ :

$$
S_{00}(\hat{f})=\frac{\mu / \pi\left[\hat{f}^{4} q+\hat{f}^{2}\left(\mu_{1}^{2} q+\mu_{1} \mu q_{1}+\mu_{2}^{2} q+\mu_{2} \mu q_{2}\right)+\mu_{1}^{2} \mu_{2}^{2} q+\mu_{1}^{2} \mu_{2} \mu q_{2}+\mu_{1} \mu_{2}^{2} \mu q_{1}\right]}{\hat{f}^{6}+\hat{f}^{4}\left(\mu_{1}^{2}+\mu_{2}^{2}+\mu^{2}-2 \mu \alpha\right)+\hat{f}^{2}\left(\mu_{1}^{2} \mu_{2}^{2}+\mu_{1}^{2} \mu^{2}+2 \mu_{1} \mu^{2} \alpha+\mu_{2}^{2} \mu^{2}-2 \mu_{2}^{2} \mu \alpha+\mu^{2} \alpha^{2}\right)+\mu_{2}^{2} \mu^{2}\left(\alpha+\mu_{1}\right)^{2}} .
$$


[1] H. M. Jaeger, S. R. Nagel, and R. P. Behringer, Rev. Mod. Phys. 68, 1259 (1996).

[2] N. V. Brilliantov and T. Pöschel, Kinetic Theory of Granular Gases (Oxford University Press, 2004).

[3] B. Andreotti, Y. Forterre, and O. Pouliquen, Granular Media (Cambridge University Press, 20013).

[4] A. Baule, F. Morone, H. J. Herrmann, and H. A. Makse, Rev. Mod. Phys. 90, 015006 (2018).

[5] C. S. Campbell, Annu. Rev. Fluid Mech. 22, 57 (1990).

[6] A. Puglisi, Transport and Fluctuations in Granular Flu$i d s$ (Springer-Verlag, 2015).

[7] P. Eshuis, K. van der Weele, D. van der Meer, R. Bos, and D. Lohse, Phys. Fluids 19, 123301 (2007).

[8] G. Pontuale, A. Gnoli, F. V. Reyes, and A. Puglisi, Phys. Rev. Lett. 117, 098006 (2016).

[9] J. J. Brey, J. W. Dufty, C. S. Kim, and A. Santos, Phys. Rev. E 58, 4638 (1998).

[10] R. Soto, Kinetic Theory and Transport Phenomena (Oxford University Press, 2016).

[11] C. Cercignani, R. Illner, and M. Pulvirenti, The Mathematical Theory of Dilute Gases (Springer, 1994).

[12] V. Garzó and J. W. Dufty, Phys. Rev. E 59, 5895 (1999).

[13] J. W. Dufty and V. Garzó, J. Stat. Phys. 105, 723 (2001).

[14] J. P. Hansen and I. R. McDonald, Theory of simple liquids (Academic Press Limited, London, 1986).

[15] D. J. Evans and G. P. Morriss, Statistical Mechanics of Nonequilibrium Liquids (Academic Press, 1990).

[16] A. Cavagna, Phys. Rep. 476, 51 (2009).

[17] W. Götze, Complex Dynamics of Glass-Forming Liquids: A Mode-Coupling Theory (Oxford University Press, 2008).

[18] W. T. Kranz, M. Sperl, and A. Zippelius, Phys. Rev. E 87, 022207 (2013).

[19] W. T. Kranz, F. Frahsa, A. Zippelius, M. Fuchs, and M. Sperl, Phys. Rev. Lett. 121, 148002 (2018).

[20] A. Gnoli, A. Lasanta, A. Sarracino, and A. Puglisi, Sci. Rep. 6, 38604 (2016).

[21] C. Scalliet, A. Gnoli, A. Puglisi, and A. Vulpiani, Phys. Rev. Lett. 114, 198001 (2015).

[22] A. Plati, A. Baldassarri, A. Gnoli, G. Gradenigo, and A. Puglisi, Phys. Rev. Lett. 123, 038002 (2019).

[23] E. Ehrichs, H. Jaeger, G. S. Karczmar, J. B. Knight, V. Y. Kuperman, and S. R. Nagel, Science 267, 1632 (1995).

[24] M. E. Möbius, B. E. Lauderdale, S. R. Nagel, and H. M. Jaeger, Nature 414, 270 (2001).

[25] N. Rivas, S. Luding, and A. R. Thornton, New J. Phys. 15, 113043 (2013).
[26] V. Folli, N. Ghofraniha, A. Puglisi, L. Leuzzi, and C. Conti, Sci. Rep. 3, 2251 (2013).

[27] A. Rabani, G. Ariel, and A. Be'er, PloS one 8 (2013).

[28] C. Chen, S. Liu, X.-q. Shi, H. Chaté, and Y. Wu, Nature 542, 210 (2017).

[29] C.-k. Tung, C. Lin, B. Harvey, A. G. Fiore, F. Ardon, M. Wu, and S. S. Suarez, Sci. Rep. 7, 1 (2017).

[30] C. Battle, C. P. Broedersz, N. Fakhri, V. F. Geyer, J. Howard, C. F. Schmidt, and F. C. MacKintosh, Science 352, 604 (2016).

[31] M. Baldovin, F. Cecconi, M. Cencini, A. Puglisi, and A. Vulpiani, Entropy 20, 807 (2018).

[32] A. Lasanta and A. Puglisi, J. Chem. Phys. 143, 064511 (2015).

[33] M. Baldovin, A. Puglisi, and A. Vulpiani, PLoS ONE 14, e0212135 (2019).

[34] D. Villamaina, A. Baldassarri, A. Puglisi, and A. Vulpiani, J. Stat. Mech. 2009, P07024 (2009).

[35] A. Sarracino, D. Villamaina, G. Gradenigo, and A. Puglisi, Europhys. Lett. 92, 34001 (2010).

[36] A. Gnoli, A. Puglisi, A. Sarracino, and A. Vulpiani, Plos One 9, e93720 (2014).

[37] V. F. Sears, Proc. Phys. Soc. 86, 953 (1965).

[38] H. D. Vollmer, Z. Physik 33, 103 (1979).

[39] A. Rahman, Phys. Rev. 136, A405 (1964).

[40] S. Plimpton, J. Comp. Phys 117, 1 (1995).

[41] H. P. Zhang and H. A. Makse, Phys. Rev. E 72, 011301 (2005)

[42] L. E. Silbert, D. Ertas, G. S. Grest, T. C. Halsey, D. Levine, and S. J. Plimpton, Phys. Rev. E 64, 051302 (2001)

[43] N. V. Brilliantov, F. Spahn, J. M. Hertzsch, and T. Pöschel, Phys. Rev. E 53, 5382 (1996).

[44] Lammps Mailing List (2019), https://lammps.sandia. gov/threads/msg80848.html.

[45] Lammps Release Review (2019), https://github.com/ lammps/lammps/releases/tag/stable_5Jun2019.

[46] A. Puglisi, A. Sarracino, G. Gradenigo, and D. Villamaina, Gran. Matt. 14, 235 (2012).

[47] R. Zwangzig, Nonequilibrium statistical mechanics (Oxford Univ. Press, 2001).

[48] C. Gardiner, Handbook of Stochastic Methods for Physics, Chemistry and the Natural Sciences (Springer- Verlag, Berlin, 1990).

[49] P. Langevin, C. R. Acad. Sci. (Paris) 146, 530 (1908), translated in Am. J. Phys. 65, 1079 (1997).

[50] I. Goldhirsch, Chaos 9, 659 (1999).

[51] L. P. Kadanoff, Rev. Mod. Phys. 71, 435 (1999). 